\title{
Modeling Behavioral Heterogeneity in Demand Theory*
}

\author{
Isabelle Maret \\ B.E.T.A/Theme, UMR 7522 du CNRS, Université Louis Pasteur, \\ 61, avenue de la Forêt Noire, 67085 STRASBOURG CEDEX, France \\ (e-mail : maret@cournot.u-strasbg.fr)
}

January 2001

\begin{abstract}
This paper is a first step in answering B. Villemeur's $(1998,1999)$ and Hildenbrand's (1998) criticism of the notions of behavioral heterogeneity introduced in demand theory by Grandmont (1992) and Kneip (1999). As in the Grandmont-Kneip approach, we define a notion of behavioral heterogeneity such that if the population is sufficiently heterogeneous, the aggregate budget share function is proved to become insensitive to changes in prices and income. However, in contrast to the aforementioned literature, this insensitivity in the aggregate is not explained by any insensitivity property at the microeconomic level, but rather by a "balancing effect" : For any commodity, the negative effect on market budget share induced after a change in prices or income by individuals who decrease their budget share is compensated by the existence of individuals who increase their budget share.
\end{abstract}

Keywords and Phrases: Aggregation; Behavioral heterogeneity; Large economy; Balancing effect; Insensitivity of maket budget shares to changes in prices and income.

JEL Classification Numbers: D11; D12; D30

\footnotetext{
${ }^{*}$ The author is very grateful to Etienne B. de Villemeur for fruitful discussions and to Pierre Dehez, Rodolphe Dos Santos Ferreira, Gaël Giraud and Hubert Stahn for their helpfull comments. She also wants to thank participants at the CORE-IRES Workshop on Aggregation of Demand 2001, in particular, Werner Hildenbrand, Reinhard John and Alois Kneip, as well as participants at the WEHIA 2001 and at seminars in Strasbourg, BETA/ Theme. The ususal caveat applies.
} 


\section{Introduction}

It is well known that restrictions on the distribution of individual demand functions imply important market demand properties which cannot be derived from assumptions on individual behaviors alone. The decisive contribution of Hildenbrand (1994) demonstrates that an assumption over the distribution of individual demand vectors provides a basis for the Law of Demand. The main support of this assumption is its accordance with empirical data. This restriction is shown to be connected with the sensitivity of aggregate demand with changes in income. More precisely, this result ensures the positive semidefiniteness in the aggregate of the income effect matrix and the Law of Demand follows by the Slutsky decomposition of the Jacobian of market demand.

This paper is more directly inspired by two other important contributions, namely Grandmont (1992) and Kneip (1999), where restrictions on the distribution of individual demand functions are interpreted as heterogeneity requirements and directly ensure a sufficient condition for the Law of Demand. More precisely, Grandmont proposes an illuminating formalization of behavioral heterogeneity by decomposing a population of households into subpopulations which satisfy a specific parametric model of demand. He then shows that sufficient heterogeneity of the resulting distribution of model parameters, measured by the flatness of the corresponding density, implies, among others, the diagonal dominance of the Jacobian of market demand, hence the Law of Demand. Kneip extends this formalization to a non-parametric setting. The idea is again to show that enough heterogeneity of behavior can explain the insensitivity of the market budget share function to changes in prices and/or income. For this purpose, he considers a well-defined metric on, say, $(\mathcal{W}, \mu)$, the probability space of households budget share functions, and he introduces a class of distance-preserving transformations $T$ on $\mathcal{W}$. The probability measure $\mu$ is then said to satisfy a "high degree" of heterogeneity if the probability of all sets $A$ and $T(A) \subset \mathcal{W}$ is extremely close, whenever $T$ is not too far from the identity mapping. ${ }^{1}$ The important result is that a "highly heterogeneous" population of households effectively admits a market budget share function approximately insensitive to changes in prices and/or income. Again, the diagonal dominance property follows and also the positive semidefiniteness of the income effect matrix.

The problem which emerges, as pointed out by B. de Villemeur $(1998,1999)$ and Hildenbrand (1998), is that it is not clear what is the precise nature of "behavioral heterogeneity" captured by the aforementioned formalism. This point may be illustrated as follows. Suppose you parameterize the space of budget share functions of your population by some number in $\mathbb{R}$. (This will be the case, for instance, if one considers, as Quah (1997), the parametric model defined as follows: If $w$ is some generating budget share function, each agent in the economy has a budget share function $w_{\alpha}$, for some $\alpha \in \mathbb{R}$ - where $w_{\alpha}(p, x)=w\left(p, e^{-\alpha} x\right)$, 
when $p$ is a price vector and $x$ an income level.) Assume, furthermore, that the transformations with respect to which one wants to test the 'heterogeneity' of the population is the collection of translations of the parameter $\alpha \in \mathbb{R}$. Claiming that the population is "highly heterogeneous" amounts to assuming that the distribution tends to be invariant with respect to this collection of translations, which means, that in the limit, the distribution of agents should converge to some "uniform' probability distribution on $\mathbb{R}$. Since, however, there is no such probability distribution on the real line, this implies that the measure towards which the distribution of characteristics is converging is a measure on the completed real line $\overline{\mathbb{R}}=\mathbb{R} \cup\{+\infty\} \cup\{-\infty\}$, whose support reduces to $\{+\infty,-\infty\}$. In particular, any compact subset of $\mathbb{R}$ is asymptotically of measure zero.

This simple example shows the essence of what is going on. Due to this concentration phenomenon, two opposing explanations of the approximate insensitivity of market budget share, obtained for an extremely heterogeneous population, might be given. The structural property might emerge from extremely heterogeneous reactions of households but also, on the contrary, from the insensitivity of almost all (approximately identical) households. In fact, the two cases emerge in Grandmont's formalism depending on the boundary behavior of the generating demand function. If this behavior is such that the associated budget share function admits limits on the boundaries of the price-income space, then as brought out by B. de Villemeur (1998) and Hildenbrand (1998) the limit probability distribution puts all its mass on Cobb-Douglas behaviors. Hence, the set of admissible budget share functions is consequently dramatically reduced. Therefore, the insensitivity of market budget share does not emerge from highly heterogeneous reactions of households but, on the contrary, from the insensitivity of almost all households to changes in prices and/or income. In this case, the notion of behavioral heterogeneity formalized in Grandmont (1992) can be related to Hildenbrand and Kneip (1999) where a finite population is said to exhibit behavioral heterogeneity if, for any price system, the percentage of households, with budget share functions sensitive to prices changes, is low. Note that this notion loses its content when the finite economy converges towards a large economy, since the limit case implies that everybody is already insensitive. B. de Villemeur (1999) shows that the concentration phenomenon might also emerge in Kneip (1999). In addition, we point out that, in Kneip's formalisation, the support of the limit probability distribution over the set of CES budget share functions is restricted to a subset of Cobb-Douglas functions.

The main message of the paper is the following : Given some conditions over the space $\mathcal{W}$ it is possible to introduce a rather large class of distancepreserving transformations such that there exist uniform conditional distributions over equivalence classes of the population and that the aggregate budget share function is exactly constant. In other words, for a perfectly heterogeneous population, the market takes on exact Cobb-Douglas properties, although no in- 
dividual adopts a Cobb-Douglas behavior. More precisely, it is proved that for a sufficiently high degree of heterogeneity market demand is approximately insensitive to changes in prices and/or income when the only rationality assumption required at the individual level is the absence of money illusion. Thus the properties of market demand, such as the Law of Demand, obtained by Kneip (1999) remain valid. The fundamental distinction is that the insensitivity property in the aggregate never follows in our set-up from the fact that, in any equivalence class (induced by a given budget share function), almost all individual are insensitive to changes in prices or income. On the contrary, the insensitivity of market budget share emerges from an increasing balancing effect; after changes in prices or income the negative effect of individuals which decrease their budget share is always compensated by the existence of individuals which increase their budget share. Note that this alternative explanation of the Law of Demand to the insensitivity property at the microeconomic level, was already present in Hicks (1953)(p.64). Hicks underlines that the property emerges in the aggregate for the excess demand function if, either the income effect is negligeable at the micro-economic level, or income effects cancel out when aggregating over buyers and sellers.

In the next section the problem is defined. In section 3, the space of admissible budget share functions (which defines the support of the probability distribution to which our theory applies) is described and the behavioral assumption (which defines the shape of the probability distribution) is introduced in section 4 . We shall be careful when relating our hypotheses to the usual understanding of a large and "heterogeneous" population. In particular, the comparison is made with Grandmont (1992) and Kneip (1999). In section 5, we establish the link between this assumption and the insensitivity of the aggregate budget share function to changes in prices and income.

\section{The problem}

Consider $^{2}$ the demand of a large population of households for $H$ commodities. Each household is described by a budget share function $w:\left(\mathbb{R}_{+}^{*}\right)^{H} \times \mathbb{R}_{+}^{*} \rightarrow \mathbb{R}_{+}^{H}$ which quantifies its budget share for the $H$ commodities as a function of the price system, $p \in\left(\mathbb{R}_{+}^{*}\right)^{H}$, and of its income, $x \in \mathbb{R}_{+}^{*}$. If $f(p, x)$ is the demand vector of the household when the price-income vector is $(p, x)$ the household budget share vector is given by

$$
w(p, x)=p \quad f(p, x) / x,
$$

where denotes the tensor product. The space of admissible budget share functions $\mathcal{W}$ gives rise to the space $\mathcal{F}$ of admissible demand functions. For every individual budget share function $w \in \mathcal{W}$ there exists a unique demand function $f \in \mathcal{F}$, and vice versa. However, as convincingly argued by Kneip (1999), in 
order to study behavioral similarity, it is much easier to work with distributions of budget share functions.

We consider a population of households with identical income. Households diverge in their demand functions hence in their characteristics affecting demand independently of prices and income. The joint distribution of household characteristics of the different households induces a distribution $\mu$ of budget share functions on $\mathcal{W}$. The assumption that all households have the same income could be relaxed. Market demand would then depend on the joint distribution of budget share functions and income. Subsequent analysis applies then to suitable subpopulations. Furthermore, one could easily prove that the properties obtained below for the aggregate demand of a given subpopulation are preserved through aggregation.

The aggregation problem consists in asking whether there exists certain restrictions on $\mathcal{W}$ and a Borel probability distribution $\mu$ such that certain properties, such as the Law of Demand, are fulfilled by the aggregate budget share function

$$
W(p, x)=\int_{\mathcal{W}} w(p, x) d \mu
$$

In other words, we want to take the space $\mathcal{W}$ itself as given, provided it belongs to a convenient class of functional spaces, and to prove that an adequate choice of the distribution of households' characteristics, which can be interpreted as representing a highly heterogeneous population, can induce per se structural properties at the market level. In this sense, we view the approach taken in this paper as quite distinct from the one adopted by B. de Villemeur (2001). There, it is argued, loosely speaking, that, given a budget share function it is always possible to construct a complementary one such that their sum satisfies the Law of Demand.

A property of interest in this literature is the Law of Demand which asserts

$$
(p-q)^{T}(F(p, x)-F(q, x))<0,
$$

for all price vectors $p$ and $q$. It is well known that this property holds if the mean Jacobian matrix, $D_{p} F(p)$, is negative definite for all price vectors $p \in\left(\mathbb{R}_{+}^{*}\right)^{H}$. However let us recall that the Jacobian of the demand function and the Jacobian of the budget share function are linked by the following relation ${ }^{3}$

$$
\mathcal{D}_{p} D_{p} F(p, x) \mathcal{D}_{p}=-x \mathcal{D}_{W(p, x)}+x D_{p} W(p) \mathcal{D}_{p},
$$

where $\mathcal{D}_{p}$ is the diagonal matrix with elements $p_{h}$, for $h=1, \ldots, H$, on the diagonal and $\mathcal{D}_{W(p, x)}$ is the diagonal matrix with elements $W_{h}(p, x)$, for $h=$ 
$1, \ldots, H$, on the diagonal. This equality allows us to deduce that if the budget share function is not particularly sensitive in the aggregate to percentage changes in prices and income, i.e.

$$
\left\{\begin{aligned}
p_{h} \frac{\partial W_{k}(p, x)}{\partial p_{h}} & \approx 0 \\
x \frac{\partial W_{k}(p, x)}{\partial x} & \approx 0
\end{aligned}\right.
$$

for all $(p, x) \in\left(\mathbb{R}_{+}^{*}\right)^{H} \times \mathbb{R}_{+}^{*}$, then the Law of Demand is valid. ${ }^{4}$ It is this insensitivity property that we are looking for in this paper. This property induces, indeed, most of the properties one could require : the Law of Demand, but also, in particular, a negative substitution effect and a positive income effect, ${ }^{5}$ and eventually the gross substitutability property which ensures the uniqueness and global stability (for the Walrasian tâtonnement) of the equilibrium of a pure exchange economy.

\section{The space of admissible budget share func- tions}

We shall consider the following space $\mathcal{W}$ of admissible budget share functions.

Assumption 1 (i) For some $\gamma>0$ and some Lebesgue measurable set $I \subset$ $[0, \gamma]^{H}$, the space $\mathcal{W}$ of admissible budget share functions is a subset of the set $\mathcal{H}(I)$ of all functions from $\left(\mathbb{R}_{+}^{*}\right)^{H} \times \mathbb{R}_{+}^{*}$ to $I$, homogeneous of degree zero in $(p, x)$. (ii) The space $\mathcal{W}$ is large enough to ensure the following requirements. First, for any $w \in \mathcal{W}$, any $(p, x) \in \Sigma_{H}^{*}$, and any $\triangle \in \Lambda$ with $(p, x)+\triangle \in \Sigma_{H}^{*}$, there exists $w^{\prime} \in \mathcal{W}$ such that $w^{\prime}(p, x)=w((p, x)+\triangle)$. Second, there exists a subset $E$ in $\mathcal{W}$ of strictly positive measure such that any function $w$ in $E$ is not constant. (iii) Finally, for all $(p, x)$ the set $\{w(p, x) \in I \mid w \in \mathcal{W}\}$ is Lebesgue measurable.

Note that Assumption (i) is traditionally made in demand theory. It holds, for example, if $\mathcal{W}$ is the set of all budget share functions generated by utility maximization with respect to the budget identity. Nevertheless, contrary to this example, the only individual rationality required by our theory is bounded budget shares and the absence of money illusion. The latter requirement was made, for example, in Grandmont (1992). However, Grandmont did not exploit this assumption to specify its behavioral heterogeneity, while here it is crucial to prove the existence of uniform conditional distributions over equivalence classes of the population, which formalize an extremely heterogeneous population. The first part of Assumption (ii) is analogous to Assumption 1(2) in Kneip (1999). It requires the set of budget share functions to be large enough in order to remain stable following perturbations in prices and income. In particular, it prevents the 
set $\mathcal{W}$ from being finite, and we can think of it as playing a role similar to the atomless hypothesis for large economies (see Hildenbrand (1974)). The second part of Assumption (ii) ensures that one excludes the non pertinent case where all households have constant budget share functions. Finally, Assumption (iii) is a non-restrictive technical assumption.

Denote by $\mathcal{A}$ the smallest $\sigma$-algebra of $\mathcal{W}$ containing all the sets of the form $A=\left\{w \in \mathcal{W} \mid w(p, x) \in J_{p, x}\right.$ for all $\left.p, x\right\}$, with $\left\{J_{p, x}\right\}_{p, x \in\left(\mathbb{R}_{+}^{*}\right)^{H} \times \mathbb{R}_{+}^{*}}$ being an arbitrary sequence of Borel subsets of $I \subset \mathbb{R}_{+}^{H}$.

Assumption 2 The distribution $\mu$ is a probability measure on the $\sigma$-algebra $\mathcal{A}$ of $\mathcal{W}$.

The connection between the properties of market demand and the distribution $\mu$ becomes more obvious if we reformulate the expression of market budget shares. For fixed $(p, x)$, the distribution $\mu$ induces a distribution $\mu_{(p, x)}$ of individual budget share vectors $w(p, x)$ on $I$. By using the latter distribution one can rewrite the expression of market budget shares in the following way

$$
W(p, x)=\int_{I} z \mu_{(p, x)}(d z) .
$$

This relationship shows that analyzing the sensitivity of market demand with respect to changes in prices and income is equivalent to considering the sensitivity of $\mu_{(p, x)}$ with respect to varying $(p, x)$. In particular, assuming the differentiability of the market demand function is equivalent to assuming differentiability of $\int_{I} z \mu_{(p, x)}(d z)$.

Assumption 3 For every continuous function $g: I \rightarrow \mathbb{R}$ the integral $\int_{I} g(z) \mu_{(p, x)}(d z)$ is continuously differentiable with respect to $p$ and $x$.

It it important to observe that the assumption that households are not victims of money illusion allows us to simplify the analysis in two ways. First, it implies that partial derivatives of the market budget share function are homogenous of degree -1 , hence for all $\lambda>0$,

$$
\left\{\begin{array}{c}
\lambda p_{h} \frac{\partial W_{k}(\lambda p, \lambda x)}{\partial p_{h}}=p_{h} \frac{\partial W_{k}(p, x)}{\partial p_{h}} \\
\lambda x \frac{\partial W_{k}(\lambda p, \lambda x)}{\partial x}=x \frac{\partial W_{k}(p, x)}{\partial x}
\end{array} .\right.
$$

Therefore, if the insensitivity property holds in the aggregate for all normalized price-income vectors $(p, x) \in \Sigma_{H}^{*}$ it also holds for any price-income vector $(p, x) \in$ $\left(\mathbb{R}_{+}^{*}\right)^{H} \times \mathbb{R}_{+}^{*}$. More precisely, to establish Eq.(5) it is sufficient to establish $D_{(p, x)} W_{k}(p, x) \cdot e_{h}$ is low for all $(p, x) \in \Sigma_{H}^{*}$, where $e_{h}$ is the vector with null elements except the h-th element which is 1 . Second, note that for a given 
$v=(p, x) \in \Sigma_{H}^{*}$, any vector $z \in \mathbb{R}^{H+1}$ can be written as a linear combination of the vector $v$ and a vector $\Delta$ in $\Lambda$. In particular, the vector $e_{h}$ can be written $e_{h}=\frac{\alpha}{\|\Delta\|} \Delta+\frac{\beta}{\|v\|} v$ where $\alpha, \beta \in \mathbb{R}, \Delta \in \Lambda$ and $\|\cdot\|$ denotes the Euclidean metric. Hence, $D_{v} W_{k}(v) \cdot e_{h}=\frac{\alpha}{\|\Delta\|} D_{v} W_{k}(v) \cdot \Delta+\frac{\beta}{\|v\|} D_{v} W_{k}(v) \cdot v$. To conclude, to prove that Eq.(5) holds, it is enough to establish that for any $k \in\{1, \ldots, H\}$

$$
D_{v} W_{k}(v) \cdot \Delta
$$

is low for all $\Delta \in \Lambda$ (since by homogeneity $D_{v} W_{k}(v) \cdot \frac{v}{\|v\|}=0$ ).

\section{The behavioral heterogeneity}

\subsection{Definition}

The fundamental issue is how to define behavioral heterogeneity. One would tend to speak of "extreme behavioral heterogeneity" if the distribution on the space $\mathcal{W}$ is close to a distribution such that "all subsets of equal size" have approximately the same probability. The problem that arises with such a requirement is that it is difficult to give a precise meaning of "all subsets of equal size" in $\mathcal{W}$. Due to this mathematical difficulty, behavioral heterogeneity has to be defined in some specific sense.

First, to give a mathematical content to the size of a subset in $\mathcal{W}, \mathcal{W}$ has to be endowed with a metric. Let us endowed $\mathcal{W}$ with the supremum norm

$$
d\left(w, w^{\prime}\right)=\sup _{(p, x) \in\left(\mathbb{R}_{+}^{*}\right)^{H} \times \mathbb{R}_{+}^{*}}\left\|w(p, x)-w^{\prime}(p, x)\right\| .
$$

Note that by homogeneity of the individual budget share functions

$$
\sup _{(p, x) \in\left(\mathbb{R}_{+}^{*}\right)^{H} \times \mathbb{R}_{+}^{*}}\left\|w(p, x)-w^{\prime}(p, x)\right\|=\sup _{(p, x) \in \Sigma_{H}^{*}}\left\|w(p, x)-w^{\prime}(p, x)\right\| .
$$

Hence, in order to study behavioral similarity on $\mathcal{W}$, one can focus on the set of normalized price-income vectors, $\Sigma_{H}^{*}$.

Second, in order to formally define heterogeneity of households with respect to a "perturbation" of the price-income vector, one has to introduce a class of distance-preserving transformations on the functional space $\mathcal{W}$. A high degree of behavioral heterogeneity is then obtained in the population if the probabilities of all sets $A, T(A) \subset \mathcal{W}$ are extremely close, whenever $T$ is a distance-preserving transformation $^{6}$ from $\mathcal{W}$ onto $\mathcal{W}$ not too far from the identity transformation $w \mapsto w$, i.e. for any given $v \in \Sigma_{H}^{*}$ and for all Borel sets $J \in I$,

$$
\mu(\{w \in \mathcal{W} \mid w(p, x) \in J\}) \approx \mu(T(\{w \in \mathcal{W} \mid w(p, x) \in J\})) .
$$


The class of distance-preserving transformations considered so far in the literature is the class of affine transformations $T_{\Delta}$ (see Grandmont (1992) and Kneip (1999)), which can be written as in Kneip (1999)

$$
\forall w \in \mathcal{W}, \forall \Delta \in\left(\mathbb{R}_{+}^{*}\right)^{H+1}, \forall(p, x) \in\left(\mathbb{R}_{+}^{*}\right)^{H+1} \quad T_{\Delta}[w](p, x)=w(\Delta \quad(p, x)) .
$$

Notice that Quah (1997) restricts himself to a smaller class of transformations called the homothetic transformations. It is important to observe that the class of affine transformations is just one of many possible classes of transformations which can be used to formalize heterogeneity of households with respect to a "perturbation" of the price-income vector. Furthermore, it is not obvious whether this class of transformations when applied to a non compact set $\mathcal{W}$ defines a behavioral heterogeneity in the sense of the aforementioned "balancing effect". This was first pointed out by B. de Villemeur (1999). The author underlines that, for $T=T_{\Delta}$ condition (11) implies that, at the limit (when the two terms are strictly equal), the set of possible individual budget share functions might be drastically restricted (the support of the probability measure is no longer the whole set $\mathcal{W}$ ). We can prove in addition that the support of the limit probability distribution over the set of CES budget share functions is restricted to a subset of Cobb-Douglas functions. Denote by $O_{w^{*}}$ the orbit of a given budget share function $w^{*} \in \mathcal{W}$, i.e. $O_{w^{*}}=\left\{w \in \mathcal{W} / \exists n \in \mathbb{Z}\right.$ such that $\left.w=T^{n}\left(w^{*}\right)\right\}$. Note that, in an extremely heterogeneous population, two budget share functions in $O_{w^{*}}$ have the same weight. This is easily seen if the subset $J$ in condition (11) is defined to be the singleton $\left\{w^{*}\right\}$. However, when $T=T_{\delta}$ nothing forbids the cardinality of any orbit to be infinite. In this case, the weight given at the limit to any compact subset of such an orbit goes to zero. Hence, for the limit probability distribution any weight given to an orbit of infinite cardinality is concentrated on its boundary elements. The boundary elements of $O_{w^{*}}$, if they exist, are defined by $w_{\text {sup }}\left(w^{*}\right)=\lim _{j \rightarrow+\infty} T^{j}\left(w^{*}\right)$ and $w_{\text {inf }}\left(w^{*}\right)=\lim _{j \rightarrow-\infty} T^{j}\left(w^{*}\right)$. In general, these boundary behaviors cannot be identified. Note, however, that when $w^{*}$ is a CES budget share function, the boundary behaviors are Cobb-Douglas behaviors. As an illustration, consider the budget share function defined in a two-commodity economy by

$$
w^{*}(p, x)=\frac{\left(a^{\sigma}\left(p_{1}\right)^{1-\sigma},(1-a)^{\sigma}\left(p_{2}\right)^{1-\sigma}\right)}{a^{\sigma}\left(p_{1}\right)^{1-\sigma}+(1-a)^{\sigma}\left(p_{2}\right)^{1-\sigma}}
$$

where $0<a<1$ and $\sigma>0$, Then, if $T=T_{\delta}$ with $\delta=(1.1,0,0)$, one has $w_{\text {sup }}\left(w^{*}\right)=(1,0)$ or $(0,1)$ and $w_{\text {inf }}\left(w^{*}\right)=(1,0)$ or $(0,1)$. To conclude, for CES behaviors, the insensitivity obtained in the aggregate for an extremely heterogeneous population as defined by Kneip (1999) does not emerge from extremely heterogeneous reactions of households but from the insensitivity of almost all (approximately identical) households. 
In this paper, we introduce new classes of distance-preserving transformations which ensures the existence of a uniform distribution over any orbit. An extremely heterogeneous population can therefore be represented by a probability measure such that the conditional distributions over the orbits are uniform. In this case, Eq. (11) formalizes now a behavioral heterogeneity in the sense of the "balancing effect" - the existence of the uniform distribution over any orbit forbids the concentration phenomenon which emerged in Kneip (1999). The following condition characterized a class of transformations, $\mathcal{T}$, to which our theory applies.

Condition 1 (i) Any transformation $T \in \mathcal{T}$ is a bijective linear mapping from $\mathcal{W}$ onto $\mathcal{W}$ which is distance-preserving. Furthermore, for all $T \in \mathcal{T}, T^{-1} \in \mathcal{T}$. (ii) For a given $(p, x) \in \Sigma_{H}^{*}$ and a given $\triangle \in \Lambda$ such that $(p, x)+\triangle \in \Sigma_{H}^{*}$ and $\|\triangle\| \in \mathbb{Q}$, there exists $T_{p, x, \Delta} \in \mathcal{T}$ such that

$$
T_{p, x, \Delta}[w](p, x)=w((p, x)+\triangle)
$$

for any $w \in \mathcal{W}$. (iii) For any $T \subset \mathcal{T}$ and any $w^{*} \in \mathcal{W}$, the orbit $\{w \in \mathcal{W} / \exists n \in \mathbb{Z}$ such that $\left.w=T^{n}\left(w^{*}\right)\right\}$ is of finite cardinality.

Condition (i) ensures that for any $A \subset \mathcal{W}$ and any $T \in \mathcal{T}$, the subsets $A$ and $T(A)$ are of equal size. Condition (ii) guarantees that the class of transformations can be used to formalize heterogeneity of households with respect to a "perturbation" of the price-income vector. Finally, Condition (iii) is the crucial restriction which ensures that there exists a probability measure over $\mathcal{W}$ such that the conditional distribution over any orbit $O_{w^{*}}$ is uniform.

Is it possible to construct an example of a non-trivial family of budget share functions in such a way that the theory developed in this paper applies ? The following example answers positively to this question.

\subsection{Example}

In the spirit of Grandmont's (1992) construction, the set of feasible budget share functions in our population, $\mathcal{W}$, is the collection of functions $\left\{w_{\alpha}\right\}_{\alpha \in \Lambda_{T} \subset \mathbb{R}^{H+1}}$ with $w_{\alpha}$ defined by :

$$
w_{\alpha}(p, x)=T_{\alpha}[\bar{w}](p, x):=\bar{w}\left(t_{\alpha}(p, x)\right)
$$

for all $(p, x) \in \Sigma_{H}^{*}$, where $\bar{w}$ is a differentiable function from $\left(\mathbb{R}_{+}^{*}\right)^{H} \times \mathbb{R}_{+}^{*}$ to $I$ homogeneous of degree zero in $(p, x)$ called the generator. For any $\alpha \in \Lambda_{T}$, the transformation $T_{\alpha}$ over $\mathcal{W}$ is defined through the transformation of the price-income vector $t_{\alpha}$. In a one-commodity economy, ${ }^{7}$ the class $\left\{t_{\alpha}\right\}_{\alpha \in \Lambda_{T}}$ where 


$$
\begin{gathered}
\Lambda_{T}=\left\{\alpha \in\left(-\frac{\sqrt{2}}{2}, \frac{\sqrt{2}}{2}\right) \mid \sqrt{2} \alpha \in \mathbb{Q}\right\} \text { and } t_{\alpha}: \Sigma_{2}^{*} \rightarrow \Sigma_{2}^{*} \text { is defined by } \\
t_{\alpha}(p, x)=\left\{\begin{array}{c}
(p+\alpha, x-\alpha) \text { if } x>\alpha, \\
\left(\alpha, \frac{\sqrt{2}}{2}-\alpha\right) \text { if } x=\alpha, \\
\left(p+\alpha-\frac{\sqrt{2}}{2}, x-\alpha+\frac{\sqrt{2}}{2}\right) \text { if } x<\alpha .
\end{array}\right.
\end{gathered}
$$

The extension of this class of transformations to an economy including any number of commodities can easily be built. The (rather long) formula is available by the author upon request and can also be found in Maret (2001).

Our population is then described by a probability distribution of $\alpha$ close to the uniform distribution over $\Lambda_{T}$. One can require without loss of generality that this population fulfills Assumptions 1 to 3. The crucial point is to establish that the class $\left\{T_{\alpha}\right\}_{\alpha \in \Lambda_{T}}$ fulfills Condition 1. To make this point clear, let us consider the whole class of transformations, $\mathcal{T}^{*}$, defined through transformations of the price-income vectors, i.e. for any $T_{t} \in \mathcal{T}^{*}$ there exists a function $t \in \Gamma^{*}$ from $\Sigma_{H}^{*}$ onto $\Sigma_{H}^{*}$ such that for any $w \in \mathcal{W}, T_{t}[w]$ is defined by

$$
T_{t}[w](p, x)=w(t(p, x)) \text { for all }(p, x) \in \Sigma_{H}^{*} .
$$

Note that when the class $\mathcal{T}^{*}$ is used to define behavioral heterogeneity of a population over a set $\mathcal{W}$, such that for any $T_{t} \in \mathcal{T}^{*}, T_{t}[w] \in \mathcal{W}, \mathcal{T}^{*}$ fulfills Condition 1 if and only if $\Gamma^{*}$ satisfies the following requirement.

Condition 2 (i) Any $t \in \Gamma^{*}$ is a bijective function from $\Sigma_{H}^{*}$ onto $\Sigma_{H}^{*}$. Furthermore, $\forall t \in \Gamma^{*}, t^{-1} \in \Gamma^{*}$. (ii) For any given $(p, x) \in \Sigma_{H}^{*}$ and any given $\triangle \in \Lambda$ with $(p, x)+\triangle \in \Sigma_{H}^{*}$ and $\|\triangle\| \in \mathbb{Q}$, there exists $t_{p, x, \Delta} \in \Gamma^{*}$ such that $t_{p, x, \Delta}(p, x)=(p, x)+\triangle$. (iii) For any $t \in \Gamma^{*}$, there exists a finite number $n \in \mathbb{N}^{*}$ such that $t^{n}(p, x)=(p, x)$ for all $(p, x) \in \Sigma_{H}^{*}$.

Condition (i) ensures Condition 1(i). Effectively, one easily checks that any transformation defined by $(17)$ is a linear mapping from $\mathcal{W}$ onto $\mathcal{W}$. Notice, then, that $T_{t}$ is a bijective mapping from $\mathcal{W}$ onto $\mathcal{W}$ as long as $t$ is a bijective function from $\Sigma_{H}^{*}$ onto $\Sigma_{H}^{*}$. Its inverse mapping is then defined by

$$
T_{t}^{-1}[w](p, x)=w\left(t^{-1}(p, x)\right) \text { for all }(p, x) \in \Sigma_{H}^{*},
$$

where $t^{-1}$ is the inverse function of $t$. Furthermore, any $T_{t} \in \mathcal{T}^{*}$ is distancepreserving since the property of definition ?? holds for $v^{T}=t^{-1}(p, x)$. Condition (ii) (respectively Condition (iii)) trivially ensures Condition 1(ii) (respectively Condition 1(iii)).

Notice that the class of affine transformations considered in the literature satisfies two conditions analogous to (i) and (ii). First, the class of affine transformations satisfies Condition (i) when $\Sigma_{H}^{*}$ has been substituted by $\left(\mathbb{R}_{+}^{*}\right)^{H} \times \mathbb{R}_{+}^{*}$. 
Second, it satisfies the following requirement equivalent to Condition (ii) : For any given $(p, x) \in \Sigma_{H}^{*}$ and any given $\triangle \in\left(\mathbb{R}_{+}^{*}\right)^{H+1}$ with $\triangle \quad(p, x) \in \Sigma_{H}^{*}$, there exists $t_{\triangle, p, x} \in \Gamma^{*}$ such that $t_{\triangle, p, x}(p, x)=\triangle \quad(p, x)$. However, it does not fulfill the crucial Condition (iii) which is introduced here to ensure, in contrast to Kneip (1999), that condition (11) generates a behavioral heterogeneity in the sense of the balancing effect.

It remains to prove that the class $\left\{t_{\alpha}\right\}_{\alpha \in \Lambda_{T}}$ fulfills Condition 2. By construction $t_{\alpha}: \Sigma_{2}^{*} \rightarrow \Sigma_{2}^{*}$ is bijective, i.e. Condition 2(i) holds. Condition 2(ii) trivially holds for $t_{p, x, \Delta}=t_{\alpha}$ and $\alpha$ such that $\left(\begin{array}{c}\alpha \\ -\alpha\end{array}\right)=\Delta$. It remains to prove that for any given $\boldsymbol{\alpha} \in \Lambda_{T}$ there exists $n \in \mathbb{N}$ such that $t_{\alpha}^{n}(p, x)=(p, x)$ for all $(p, x) \in \Sigma_{2}^{*}$. By assumption $\sqrt{2}|\alpha| \in \mathbb{Q}_{+}$, hence $\exists q \in \mathbb{N}$ such that $\frac{1}{q}=\sqrt{2}|\alpha|$. For any $(p, x) \in \Sigma_{2}^{*}$ such that $\forall r \in \mathbb{N},(p+r \alpha, x-r \alpha) \notin\left\{\left(0, \frac{\sqrt{2}}{2}\right),\left(\frac{\sqrt{2}}{2}, 0\right)\right\}$ one has,

$$
\left[t_{\alpha}\right]^{q}(p, x)=(p, x) .
$$

For any $(p, x) \in \Sigma_{2}^{*}$ such that $\exists r \in \mathbb{N}$ with $(p+r \alpha, x-r \alpha) \in\left\{\left(0, \frac{\sqrt{2}}{2}\right),\left(\frac{\sqrt{2}}{2}, 0\right)\right\}$, one has,

$$
\left[t_{\alpha}\right]^{q-1}(p, x)=(p, x) .
$$

Hence, Condition 2(iii) holds for $n=q(q-1)$ as illustrated on figure 1 for $\alpha=\frac{\sqrt{2}}{8}$.

In this example, following Grandmont (1992), the population is generated by one function through transformations of the price-income vector. However, in contrast to Grandmont, the class of transformations is no longer the class of affine transformations but a class which fulfills Condition 2. In this case, thanks to the homogeneity requirement (absence of money illusion) the population is described by a probability measure on a dense subset of a compact set. Hence, there exists a uniform distribution over this set. Therefore, the extremely heterogeneous population is described by this uniform distribution and the concentration phenomenon does not emerge in this set-up. In other words, for the extremely heterogeneous population all households react heterogeneously to changes in prices and income, in addition any type of behavior has the same probability to emerge and any non-empty compact subset of $\mathcal{W}$ has a strictly positive probability. Notice that the result obtained in this example is even stronger than the one offered by our theory, since in this example the existence of the uniform distribution is obtained over the whole space $\mathcal{W}$ and not only over each orbit (generated by a given budget share function).

Notice that individual rationality is essentially restricted to the absence of money illusion, in particular, the differentiability of the generator is not preserved through the transformations of the price-income vector, nevertheless these transformations preserve the weak axiom of revealed preferences (when required). 


\section{Behavioral heterogeneity and the insensitiv- ity property}

In this section, we prove that as the degree of behavioral heterogeneity increases in the population, market budget shares become insensitive to changes in prices and/or income. From the previous setting, we deduce that for any given priceincome vector $(p, x)$ a high degree of behavioral heterogeneity of $\mu$ implies that for all Borel sets $J \subset I$,

$$
\begin{aligned}
\mu_{(p, x)+\Delta}(J) & =\mu(\{w \in \mathcal{W} \mid w((p, x)+\Delta) \in J\}) \\
& =\mu\left(\left\{w \in \mathcal{W} \mid T_{p, x, \Delta}(w)(p, x) \in J\right\}\right) \\
& =\mu\left(T_{p, x, \Delta}^{-1}(\{w \in \mathcal{W} \mid w(p, x) \in J\})\right) \\
& \approx \mu(\{w \in \mathcal{W} \mid w(p, x) \in J\})=\mu_{(p, x)}(J),
\end{aligned}
$$

at least if $\Delta \in \Lambda$ is not too far from $\mathbf{0}=(0,0, \ldots, 0)^{\prime}$ and $\|\Delta\| \in \mathbb{Q}$. Therefore, we get a high degree of "pointwise heterogeneity" in the sense of Kneip : For any subset $J \subset I$ the probability that a household of the population possesses a budget share function with $w(p, x) \in J$ is approximately equal to the probability that a household possesses a budget share function which takes values in $J$ at $(p, x)+\triangle$. Relation (21) expresses the fact that a high degree of behavioral heterogeneity induces a weak sensitivity of the distribution $\mu_{(p, x)}$ with respect to changes in prices and income. Let $C(I,[0,1])$ denote the space of all continuous functions from $I$ into $[0,1]$. As a consequence, a high degree of behavioral heterogeneity induces a small coefficient, $h(\mu)$, defined by

$$
h(\mu)=\max _{h=1, \ldots, H+1} \sup _{v} \sup _{g \in C(I,[0,1])}\left|\frac{\partial}{\partial \lambda}\left(\int_{I} g(z) \mu_{(p, x)+\lambda \Delta}(d z)\right)\right|_{\lambda=\mathbf{0}} \mid .
$$

A direct consequence is that market budget shares are not very sensitive to changes in prices and income.

Proposition 1 Under Assumptions 1 to 3, as the degree of behavioral heterogeneity in the population increases, market budget shares become insensitive to percentage changes in prices and/or income, i.e. for $h(\mu)$ small enough Eq. (5) holds.

Proof. For every $(p, x) \in \Sigma_{H}^{*}$ and every $\triangle \in \Lambda$ with $(p, x)+\Delta \in \Sigma_{H}^{*}$ and $\|\triangle\| \in \mathbb{Q}$, one has

$$
\left|D_{v} W_{\mu ; k}(v) \cdot \Delta\right|=\left|\frac{\partial}{\partial \lambda}\left(\int_{\mathcal{W}} w_{k}((p, x)+\lambda \triangle) d \mu\right)\right|_{\lambda=0} \mid .
$$


From Assumption 14, we deduce that

$$
\left|D_{v} W_{\mu ; k}(v) \cdot \Delta\right|=\left|\frac{\partial}{\partial \lambda}\left(\int_{\mathcal{W}} z \mu_{(p, x)+\lambda \triangle}(d z)\right)\right|_{\lambda=0} \mid \quad h(\mu),
$$

for any $\triangle \in \Lambda$ with $(p, x)+\Delta \in \Sigma_{H}^{*}$ and $\|\triangle\| \in \mathbb{Q}$. As a consequence if $h(\mu)$ is small then $\left|D_{v} W_{\mu ; k}(v) \cdot \Delta\right|$ is small $\forall \triangle \in \Lambda$ with $\|\triangle\| \in \mathbb{Q}$ and by continuity of $\left|D_{v} W_{\mu ; k}(v) \cdot \Delta\right|$ with respect to $\Delta$, this holds for all $\triangle \in \Lambda$.

This proposition implies that the structural properties of market demand obtained in Kneip (1999)(Theorems 4.2., 4.3 and 4.4), in particular, the Law of Demand over the whole set of price-income vectors, remain valid under our behavioral heterogeneity requirement. When applied to a pure exchange economy, this result also implies the gross substitutability property which ensures the uniqueness and global stability (for the Walrasian tâtonnement) of the price equilibrium (see Grandmont (1992)).

Notice that nowhere do we assume that the budget share functions are continuous or that each individual budget constraint is satisfied. Nor need the weak axiom of revealed preferences (WARP) be satisfied at any level. This shows that heterogeneity of possibly extremely irregular and irrational behaviors may, on its own, generate an extremely regular mean outcome.

\section{Conclusion}

Our notion of behavioral heterogeneity forbids that as the degree of heterogeneity increases the weight given to a subpopulation (the orbit of a given budget share function) is concentrated on its boundary elements. However, since any subpopulation might have a measure zero, this result does not prevent the concentration phenomenon over the whole space $\mathcal{W}$. The answer to this issue is to be given by further research. More precisely, one has to develop a theory which ensures the existence of a uniform distribution over the space of feasible budget share functions (as in the above example). However, in our theory even when the concentration phenomenon over a subset of subpopulations occurs, the insensitivity in the aggregate is never explained by any insensitivity at the individual level but rather by the "balancing effect" since in any subpopulation all households are sensitive to changes in prices and income.

\section{References}

B. de Villemeur, E. (1998), Heterogeneity and Stability : Variations on Scarf's Processes, Working Paper ECO No. 98/38, European University Institute, Florence. 
B. de Villemeur, E. (1999), Aggregation of Demand and Distribution of Characteristics : A Difficulty in Modelling Behavioral Heterogeneity, Working Paper No. 99/38, Université de Cergy-Pontoise, Paris.

B. de Villemeur, E. (2001), Behavioural Complementarity (not Heterogeneity) causes the Law of Demand', Working-Paper 20.14.545, Gremaq, Université de Toulouse.

Grandmont, J.M. (1992), Transformations of the Commodity Space, Behavioral Heterogeneity and the Aggregation Problem, Journal of Economic Theory 57, 1-35.

Hicks, J.R. (1953), Value and Capital, third edition, Oxford, Clarendon Press.

Hildenbrand, K. (1998), On J.M. Grandmont's Modelling of Behavioral Heterogeneity, Discussion Paper No. A-580, SFB 303, Universität Bonn.

Hildenbrand, W. (1974), Core and Equilibria of a Large Economy, Princeton, Princeton University Press.

Hildenbrand, W. (1994), Market Demand: Theory and Empirical Evidence, Princeton, Princeton University Press.

Hildenbrand W. and A. Kneip (1999), On Behavioral Heterogeneity, Discussion Paper No. A-589, SFB 303, Universität Bonn.

Kneip, A. (1999), Behavioral Heterogeneity and Structural Properties of Aggregate Demand, Journal of Mathematical Economics, Vol. 31, pp. 49-79.

Maret, I. (2001), Modeling Behavioral Heterogeneity in Demand Theory, Working Paper BETA n ${ }^{\circ}$ 2001-04, Université Louis Pasteur, Strasbourg.

Quah, J. (1997), The Law of Demand when Income is Price Dependent, Econometrica, Vol. 65, $\mathrm{n}^{\circ}$ 6, pp. 1421-1442. 
Figure 1 : An example of a class of transformations for $H=1$.

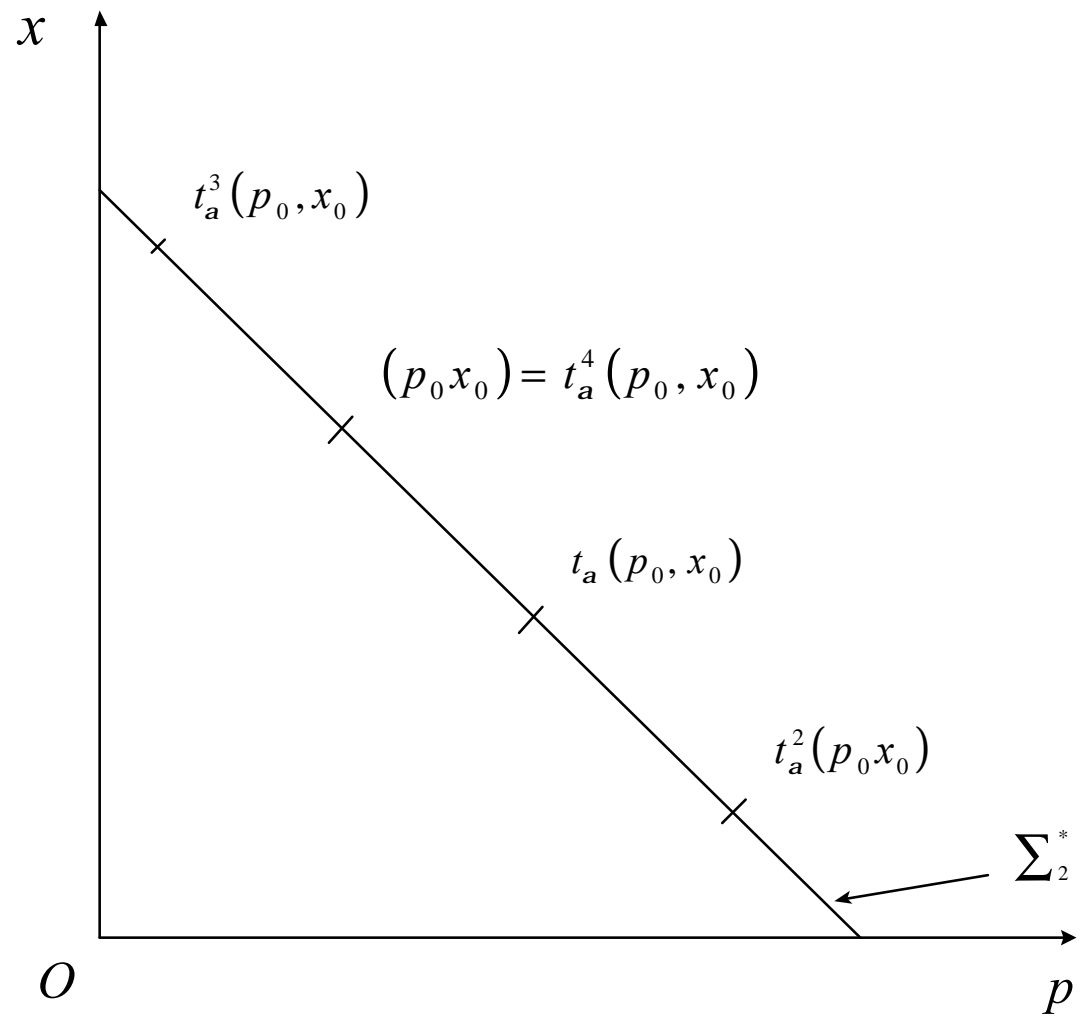




\section{FOOTNOTES}

1. Note that Grandmont's (1992) parametric population enters in this framework, in this case heterogeneity is tested with respect to the collection of translations over the parameter set.

2. Let us introduce the following notations. Denote by $v=(p, x)$, a price-income vector, with $v_{H+1}=x$, by $e \in \mathbb{R}^{H+1}$ the vector $(1,1, \ldots, 1)$. The vector with elements $1 / a_{1}, \ldots, 1 / a_{L}$ is written $a^{-1}$. In addition, we denote by $\Sigma_{H}^{*}$ and by $\Lambda$ the following sets,

$$
\begin{aligned}
\Sigma_{H}^{*} & =\left\{(p, x) \in\left(\mathbb{R}_{+}^{*}\right)^{H} \times \mathbb{R}_{+}^{*} \mid \sum_{h=1}^{H} p_{h}+x=\frac{\sqrt{2}}{2}\right\} \\
\text { and } \Lambda & =\left\{\Delta \in \mathbb{R}^{H+1} \mid \sum_{h=1}^{H+1} \Delta_{h}=0\right\} .
\end{aligned}
$$

Finally, for any bijective mapping $T: \mathcal{W} \rightarrow \mathcal{W}$ and any integer $n, T^{n}$ stands for $T \circ \ldots \circ T$, the $n^{\text {th }}$ composition of $T$ with itself.

3. In addition, one has

$$
\mathcal{D}_{p} D_{x} F(p, x)=W(p, x)+x D_{x} W(p, x) .
$$

4. Nevertheless, to deduce this property, one has to add the assumption that there exists a constant $c>0$ such that $\int_{\mathcal{W}} w_{h}(p, x) d \mu \geq c$ for all $p, x$ and $h=1, \ldots, H$ (see [8] Theorem 4.2). Note that this additional assumption could be weaken to hold only on a compact price set since for many purposes it is enough to obtain the Law of Demand on a compact price set.

5. First, under the additional assumption that there exists a constant $c^{*}>0$ such that $\lambda_{\min }\left(M^{(1)}(p)\right) \geq c^{*}$ for all $p$, the insensitivity property implies that the aggregate matrix of income effect is positive definite for all price vectors $p \in\left(\mathbb{R}_{+}^{*}\right)^{H}$. Second, under the additional assumption that there exists a constant $c^{* *}>0$ such that $\int_{\mathcal{W}} w_{h}(p, x) w_{k}(p, x) d \mu \geq c^{* *}$ for all $h, k$ and every $p, x$, it ensures that the aggregate Slutsky matrix is negative semi-definite for all price vectors $p \in\left(\mathbb{R}_{+}^{*}\right)^{H}$ (see [8] Theorems 4.3 and 4.4).

6. Denote by $\mathcal{H}\left(\left(\mathbb{R}_{+}^{*}\right)^{H}\right)$ the space of all homogeneous functions of degree zero from $\left(\mathbb{R}_{+}^{*}\right)^{H+1}$ into $\left(\mathbb{R}_{+}\right)^{H}$. A distance-preserving transformation over this space is defined by the following.

Definition 1 A bijective linear mapping $T: \mathcal{H}\left(\left(\mathbb{R}_{+}^{*}\right)^{H}\right) \rightarrow \mathcal{H}\left(\left(\mathbb{R}_{+}^{*}\right)^{H}\right)$ is called a distance-preserving transformation, if for any $v \in \Sigma_{H}^{*}$, there exists a 
unique $v_{T} \in \Sigma_{H}^{*}$ such that $\left\|w(v)-w^{\prime}(v)\right\|=\left\|w^{T}\left(v_{T}\right)-w^{T}\left(v_{T}\right)\right\|$ holds for all $w, w^{\prime} \in \mathcal{H}\left(\left(\mathbb{R}_{+}^{*}\right)^{H}\right)$ and $w^{T}=T(w), w^{T}=T\left(w^{\prime}\right)$.

It is easily verified that any such transformation preserves distances in the following sense : For all $w, w^{\prime} \in \mathcal{H}\left(\left(\mathbb{R}_{+}^{*}\right)^{H}\right)$

$$
d\left(w, w^{\prime}\right)=d\left(T(w), T\left(w^{\prime}\right)\right)
$$

7. Note that an analogous two-dimensional set-up could be used to formalize, in a two-commodity economy, heterogeneous reactions of households to price changes, for a fixed income level $\bar{x}$. In this case, the set of normalized price-income vector $\Sigma_{2}^{*}$ is substituted by

$$
\Sigma_{2, \bar{x}}^{*}=\left\{(p, x) \in\left(\mathbb{R}_{+}^{*}\right)^{2} \times \mathbb{R}_{+}^{*} \mid p_{1}+p_{2}=\frac{\sqrt{2}}{2} \text { and } x=\bar{x}\right\} .
$$

Then, the class $\left\{t_{\alpha}\right\}_{\alpha \in \Lambda_{T}}$ where $t_{\alpha}: \Sigma_{2, \bar{x}}^{*} \rightarrow \Sigma_{2, \bar{x}}^{*}$ is defined by

$$
t_{\alpha}\left(p_{1}, p_{2}, \bar{x}\right)=\left\{\begin{array}{c}
\left(p_{1}+\alpha, p_{2}-\alpha, \bar{x}\right) \text { if } p_{2}>\alpha \\
\left(\alpha, \frac{\sqrt{2}}{2}-\alpha, \bar{x}\right) \text { if } p_{2}=\alpha \\
\left(p_{1}+\alpha-\frac{\sqrt{2}}{2}, p_{2}-\alpha+\frac{\sqrt{2}}{2}, \bar{x}\right) \text { if } p_{2}<\alpha
\end{array}\right.
$$

